\title{
ATYPICAL MANIFESTATION OF PRIMARY SYNOVIAL CHONDROMATOSIS IN TEENAGE FEMALE
}

Caio Alexandre Zanoni', Sarah Polyane Silva Veloso', Agatha Siqueira Afonso' ${ }^{1}$, Marlon Sousa Lopes ${ }^{1}$, Regiane Duque Minardi Neves ${ }^{1}$, Marne Rodrigues Pereira Almeida', Cristina Ribeiro Medeiros de Magalhães ${ }^{1}$, Simone de Oliveira Alves ${ }^{1}$, Maria Custódia Machado Ribeiro",*, Aline Garcia Islabão

1. Hospital da Criança de Brasília José Alencar, Brasília (DF), Brazil.

*Corresponding author: mcustodiamr@gmail.com

\section{BACKGROUND}

Synovial chondromatosis is a benign condition characterized by synovial proliferation and metaplasia with development of cartilaginous or osteocartilaginous nodules within a joint, bursa or tendon sheath. The disease is commonly monoarticular with the knee most often affected. However, involvement of smaller joints, such as the temporomandibular and the facet joints of the vertebral column, occasionally occurs. A marked male predominance is seen and the patients are usually young adults. This case report is about a teenage female with left shoulder affected.

\section{CASE REPORT}

An 11-year-old female patient started to experience pain in the lateral region of both feet, in the thoracic spine and shoulders, bilaterally, during movement associated with crackling and functional limitation due to pain. It was unrelated to trauma and initiated four months ago. She reported waking up with a painful condition and six-hour morning stiffness. It showed improvement after using ibuprofen without compromising other activities of daily living. In a complementary diagnostic investigation, she presented radiography of the left shoulder showing the contours of the irregular left glenohumeral joint surfaces with several nodules of ossified aspect (Fig. 1). The lesion was at the topography of the axillary and scapular recesses thus suggesting primary synovial osteochondromatosis. On physical examination, she presented pain on movement of the shoulder in all planes without other signs of inflammation. Moreover, pain on palpation through the entire segmental spine, scoliosis and a Schöber's test $20.2 \mathrm{~cm}$.

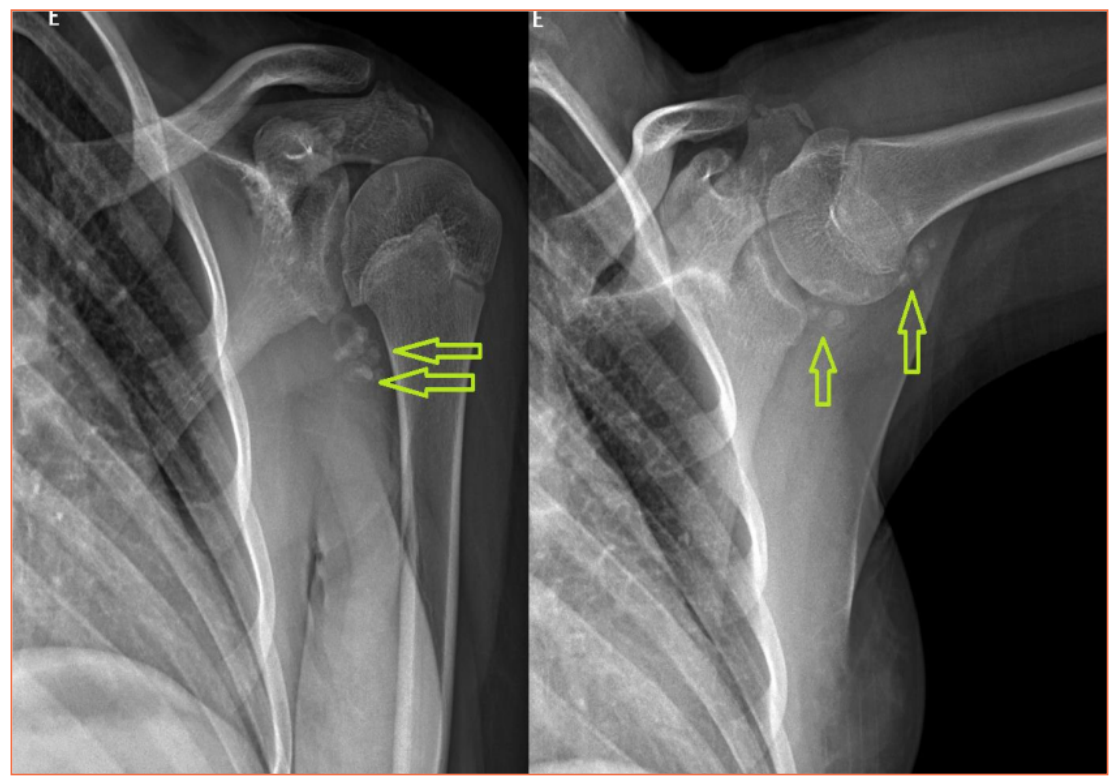

Figure 1. Radiographs of the left shoulder showing presence of calcified nodules - osteochondromas (arrows). 


\section{CONCLUSION}

This case report comes to show how important are the clinical history, anamnesis and physical examination in assessing differential diagnoses of other aetiologies of chondromatosis. Synovial osteochondromatosis may occur in the shoulder within the glenohumeral joint and its recesses and in the subacromial-deltoid bursa. Such condition can be identified either by radiography, ultrasonography or magnetic resonance imaging showing typical features according to each method. Radiography commonly shows ring-shaped calcified cartilages and periarticular soft tissues swelling with erosion of joint margins. The diagnosis may be achieved only on the basis of typical imaging findings which depending on the stage of the disease may correspond to synovial membrane proliferation, presence of cartilaginous nodules (chondromas) and calcified or ossified nodules (osteochondromas). 